\title{
Study on Formation Mechanism of Defects in Monoclinic KLu($\left(\mathrm{WO}_{4}\right)_{2}$ Crystals
}

\author{
Kunpeng Wang, Jinzhi Fang \\ National Center for Materials Service Safety, University of Science and Technology Beijing, Beijing, China. \\ Email: kpwang@ustb.edu.cn
}

Received December 23 ${ }^{\text {rd }}, 2010$; revised January 20 ${ }^{\text {th }}, 2011$; accepted May $17^{\text {th }}, 2011$.

\begin{abstract}
The laser host crystals of $\mathrm{KLu}\left(\mathrm{WO}_{4}\right)_{2}(\mathrm{KLuW})$ with large sizes up to $43 \times 35 \times 16 \mathrm{~mm}^{3}$ have been grown along [1]11], [110], b, and c crystallographic directions, respectively, by the top-seeded solution growth (TSSG) slow-cooling method. The macro defects are observed using optical microscopy. The main defects found were cracks, inclusions, growth striations, sector boundaries and twin boundaries. The formation mechanism and approaches to reduce or eliminate the defects have been analyzed.
\end{abstract}

Keywords: Growth Defects, $\mathrm{KLu}\left(\mathrm{WO}_{4}\right)_{2}$ Crystals, Top-Seeded Solution Growth

\section{Introduction}

Double tungstates containing potassium and rare-earth ions have been extensively investigated because of their applicability in diode-pumped solid-state lasers. Crystals such as $\mathrm{Nd}: \mathrm{KGd}\left(\mathrm{WO}_{4}\right)_{2}$ [1,2], Yb:KY( $\left(\mathrm{WO}_{4}\right)_{2}$ [3], and $\mathrm{KYb}\left(\mathrm{WO}_{4}\right)_{2}$ [4] have been recently used as laser materials or Raman shifters. $\mathrm{KLu}\left(\mathrm{WO}_{4}\right)_{2}$, which has $\mathrm{KGd}\left(\mathrm{WO}_{4}\right)_{2}$ related structure, is also an attractive laser host material and has found application in a wide range of optoelectronic devices $[5,6]$. More recently KLuW crystals with large sizes and good optical quality have been grown by TSSG method in our group [7]. The lattice constants were determined by using a Four-Circle Diffractometer to be $a=10.591(5) \AA, b=10.244(6) \AA, c=7.500(3) \AA$ and $\beta=130.73(2)^{\circ}$, which are slightly smaller than those of KGW [8]. The experimentally grown crystal shapes developed along different crystalline directions were compared to the predicted habits, and the influences of seed orientations on the crystal growth habits, quality and utilization ratio are analyzed by BFDH law and PBC' theory [7]. The anisotropy thermal properties were carefully studied [9].

In the present work, the crystal defects are observed and analyzed using optical microscopy. Their formation mechanism and the approach to abate and even eliminate these defects are presented.

\section{Experiments}

Single crystals of KLuW with different seed orientations have been grown by TSSG method and as an example the as-grown KLuW crystal with seed orientations along $b$ directions is shown in Figure 1. Three wafers parallel to the (110), (200), and (020) facets, respectively, are cut from the crystal and the natural grown planes are cleaned by absolute alcohol. The Opton polarizing microscope is used to observe the morphologies of (110), (200), and (020) facets and all pictures were taken by a video camera.

\section{Results and Discussions}

\subsection{Cracking and Inclusions}

Cracks have been observed in crystals with seed orientations along [111], $b$, and [110] directions, respectively. Whereas crystals grown along $c$ directions have good optical quality and no clear cracks could be observed. As shown in the Figure 1, the directions of the cracks are mostly parallel to $b$ and $(a+c)$ directions. We suggest that when the cooling rate is very fast the anisotropic thermal expansion coefficients of KLuW crystals results in cracks along $b$ and $(a+c)$ directions. In addition, the local crystal lattices are distorted greatly by the solution, impurity or gaseous solutions and therefore mechanical stresses are induced. At the same time, the thermal expansion coefficients of the inclusions are also different from those of the crystal, which also results in cracks. So understanding the forming mechanisms of inclusions is also an important issue for crystal researchers. Various kinds of inclusions have been observed in the KLuW 


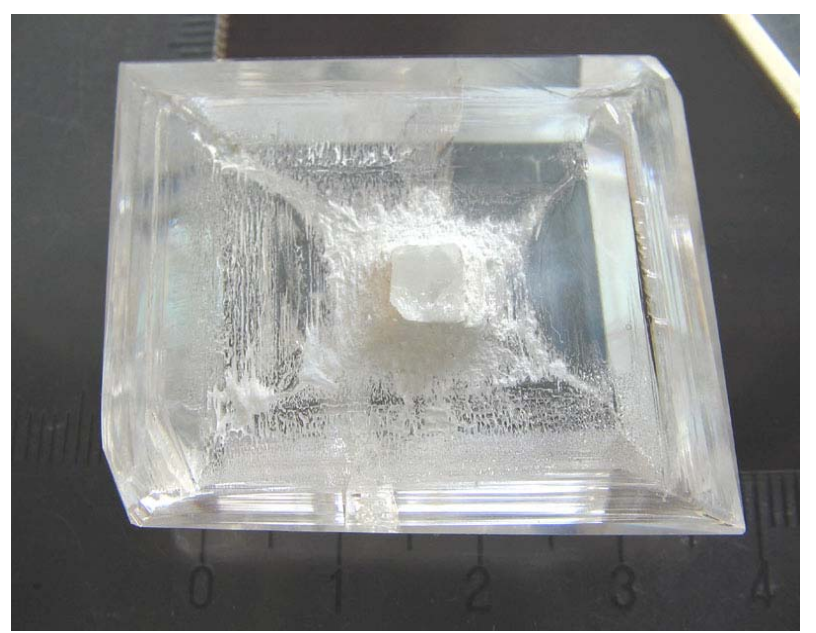

Figure 1. The as-grown KLuW crystal with seed orientations along $b$ directions and severe cracks along $a$ and $(a+c)$ directions.

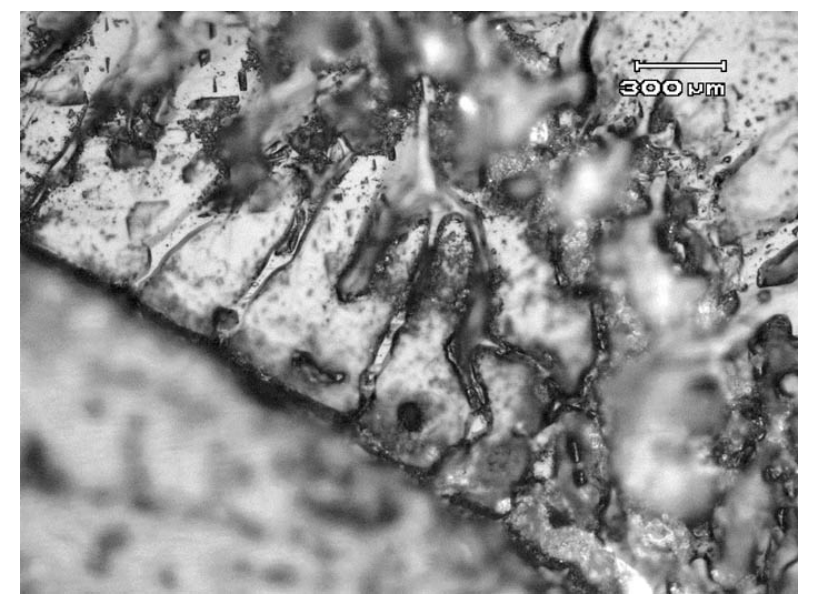

(a)

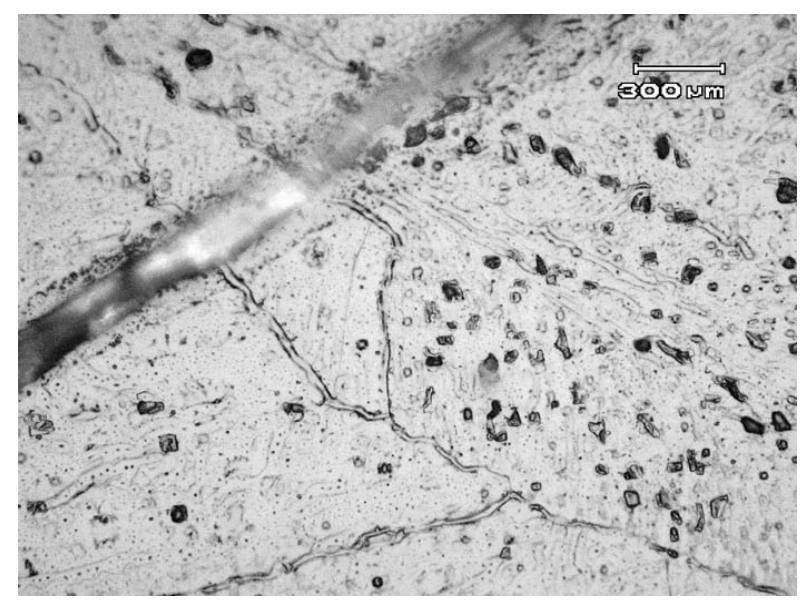

(c) crystal. Figure 2(a) shows the melt inclusions observed at the edge of the (110) face and we can see that they are large size up to hundreds of microns in length. They may be caused by the rapid cooling-rate during the last stage of crystal growth, the segregation phases formed in the system, or the multi-steps' meeting in the developing process. In addition, we suggest that the negative crystal, which is formed by the negative growth, has been formed in the melt inclusion shown in Figure 2(b) due to its regular morphology as the same of the crystal. On the other hand, the inclusions can also be caused by the impurities and insoluble particles. Figure 2(c) shows the inclusions containing insoluble particles with micrometer scale observed on the (200) faces. They often result in the step bunching and so high-purity materials should be selected to avoid impurities and insoluble particles. The inclusions described above are often symbiotic in the crystal during crystal growth. However, when the temperature

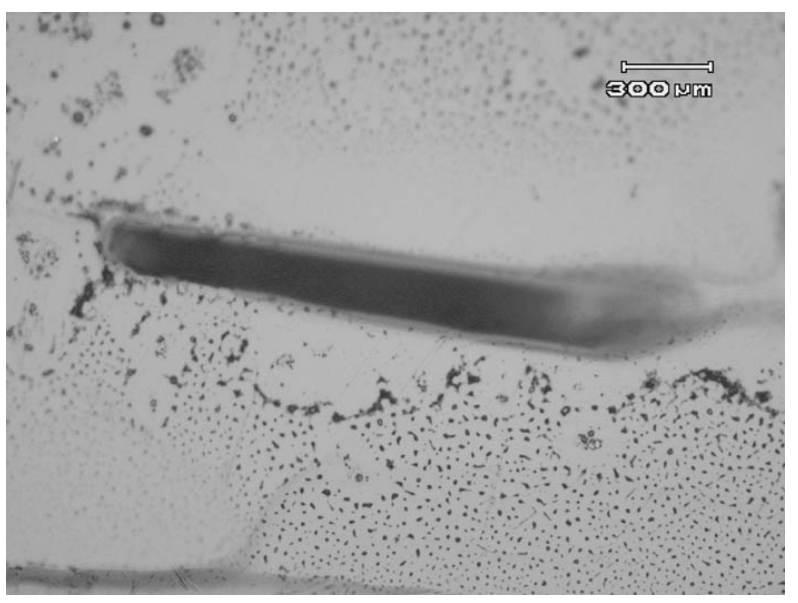

(b)

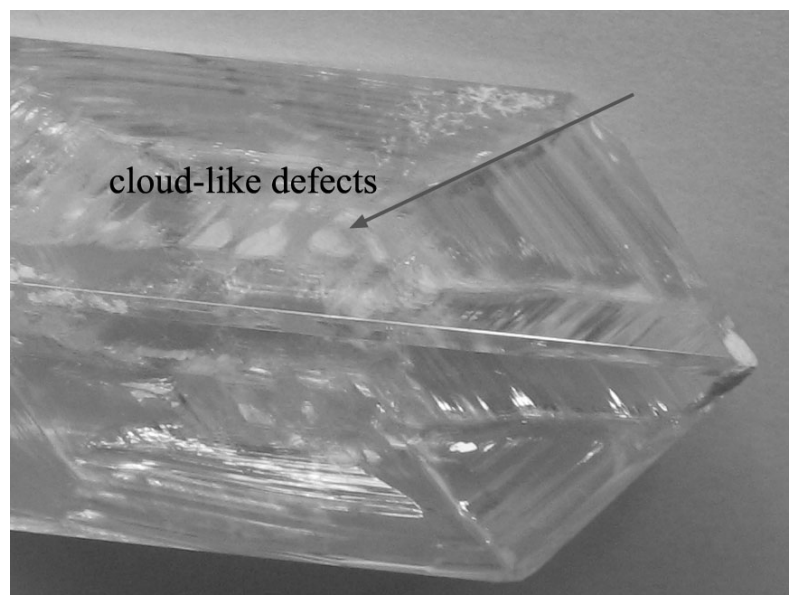

(d)

Figure 2. (a) The melt inclusions observed at the edge of the (110) face, (b) the negative crystal formed in the melt inclusion, (c) the inclusions containing insoluble particles with micrometer scale observed on the (200) faces, (d) cloud-like defects caused by large quantity of inclusions. 


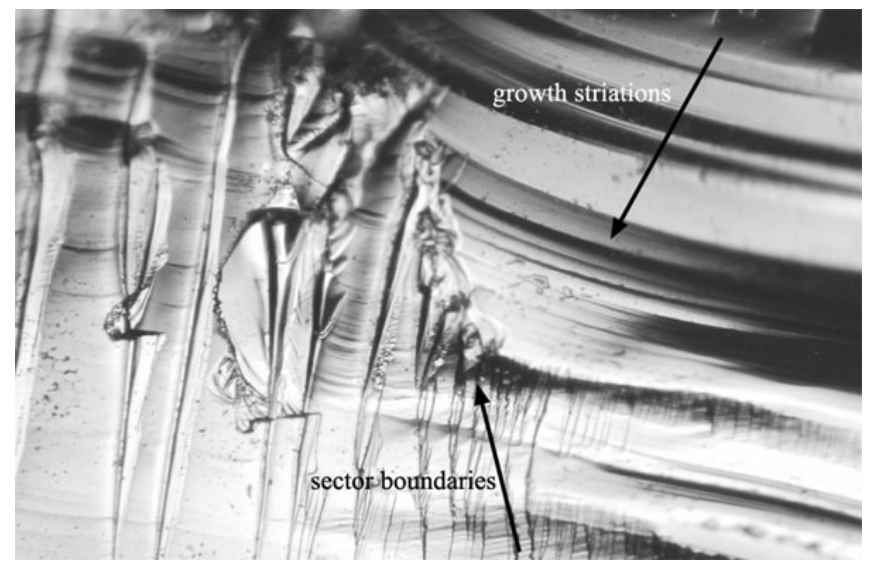

(a)

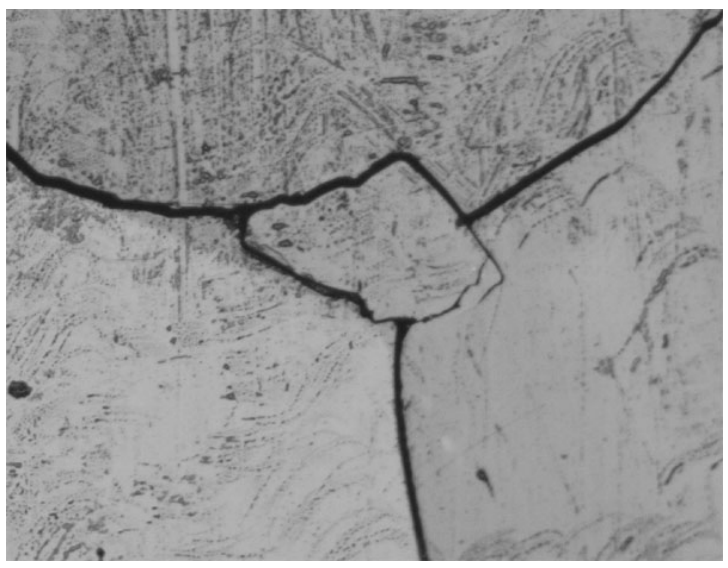

(b)

Figure 3. (a) The growth striations, sector boundaries and insoluble solid inclusions that are observed on the (110) face, (b) the twin boundaries formed on the (110) face.

fluctuates greatly, it will increase the inclusion density and cause cloud-like defects shown in Figure 2(d). The amount of cloud-like defects is related to the temperature perturbation and the cloud-like defects can usually be alleviated by annealing the as-grown crystals at proper temperature. As described in our former literature [7], the crystals grown along [1]11], $b$, and [110] directions are often poor due to the macro defects appear and however no macroscopic defects such as cracks, clouds and scattering particles were detected under the irradiation of a 5 $\mathrm{mW}$ green laser beam when grown along $c$ directions.

\subsection{Growth Striations and Sector Boundaries}

As analyzed in our former literature [7], the (110), (020), (111) faces are parallel to two or more shortest PBC's and so they are well developed. (200) and (20) are the main growth faces due to their higher attached energy. The difference of growth rates between these two faces results in structure mismatches and lattice distortion at the interface and forms sector boundaries. Impurities can be easily captured on the sector boundaries, which results in the formation of inclusions. The growth kinetics factors and the undulation of the growth conditions result in growth striations. Figure 3(a) shows the growth striations, sector boundaries and insoluble solid inclusions that are observed on the (110) face.

\subsection{Twin Boundary}

Twins might form as "accidents" during the growth of a crystal and can also develop after a crystal has grown during polymorphic transformation or during deformation. According the Hurl model [5,6], twins always generate on the edge facets which are anchored to the three-phase boundary (TPB) at the solid-liquid interface during the crystal growth. The nucleation free energy is low and the step's absorption energy is large at the TPB. So the twins will energetically favor nucleation at the TPB and impurities are easy to be absorbed at the TPB. When the steps absorb impurities, the position and direction of all future growth will be altered from the normal orientation. Figure 3(b) shows the formed twins.

\subsection{Methods of Reducing Defects}

1) Optical quality KLuW crystals can be easier obtained with seed orientations along $\mathrm{c}$ directions than along other directions.

2) Raw materials should be prepared and purified in contamination-free environment and then fully mixed.

3) High-quality seeds must be selected to avoid the extension of the dislocations to the crystal.

4) Keeping suitable growth rate and reducing temperature fluctuation help in decreasing the crystal thermal stress.

\section{Conclusions}

KLuW crystals have been successfully grown by TSSG method. Macro defects have been observed using optical microscopy. The main defects found were cracks, inclusions, growth striations, sector boundaries and twin boundaries. Methods of reducing and eliminating the defects have been discussed.

\section{Acknowledgements}

Supported by Program for New Century Excellent Talents in University (NCET-08-0722).

\section{REFERENCES}

[1] Y. Kalisky, L. Kravchik and C. Lbbe, "Repetitive Modulation and Passively Q-Switching of Diode-Pumped Nd-KGW Laser," Optics Communications, Vol. 189, No. 
1-3, 2001, pp. 113-119. doi:10.1016/S0030-4018(01)01018-5

[2] N. S. Ustimenko and A.V. Gulin, "New Self-Frequency Converted Nd:KGd( $\left(\mathrm{WO}_{4}\right)_{2}$ Raman Lasers,” Quantum Electron, Vol. 32, No. 3, 2002, pp. 229-235. doi: 10.1070/QE2002v032n03ABEH002168

[3] A. A. Demidovich, A. N. Kuzmin, G. I. Ryabtsev, M. B. Danailov, W. Strek and A. N. Titov, "Influence of Yb Concentration on Yb:KYW Laser Properties,” Journal of Alloys and Compounds, Vol. 300-301, No. 1, 2000, 238241. doi:10.1016/S0925-8388(99)00777-X

[4] M. C. Pujol, X. Mateos, R. Sole, J. Masson, J. Gavalda, X. Solans, F. Diaz and M. Augilo, "Structure, Crystal Growth and Physical Anisotropy of $\mathrm{KYb}\left(\mathrm{WO}_{4}\right)_{2}$, a New Laser Matrix," Journal of Applied Crystallography, Vol. 35, No. 1, 2002, pp. 108-114. doi:10.1107/S0021889801019914

[5] X. Mateos, V. Petrov and M. Aguilo, "Continuous-Wave Laser Oscillation of $\mathrm{Yb}^{3+}$ in Monoclinic $\mathrm{KLu}\left(\mathrm{WO}_{4}\right)_{2}$," IEEE Journal of Quantum Electronics, Vol. 40, No. 8,
2004, pp. 1056-1059. doi:10.1109/JQE.2004.831614

[6] U. Griebner, J. H. Liu, S. Rivier, A. Aznar, R. Grunwald, R. Maria, M. Aguilo, F. Diaz and V. Petrov, "Laser Operation of Epitaxially Grown $\mathrm{Yb}: \mathrm{KLu}\left(\mathrm{WO}_{4}\right)_{2}-\mathrm{KLu}\left(\mathrm{WO}_{4}\right)_{2}$ Composites with Monoclinic Crystalline Structure," IEEE Journal of Quantum Electronics, Vol. 41, No. 3, 2005, pp. 408-414. doi:10.1109/JQE.2004.842313

[7] K. P. Wang, J. X. Zhang and J. Y. Wang, "Predicted and Real Habits of Flux Grown Potassium Lutetium Tungstate Single Crystals," Crystal Growth \& Design, Vol. 5, No. 4, 2005, pp. 1555-1558. doi:10.1021/cg050019j

[8] L. I. Yudanova, O. G. Potapova and A. A. Pavlyuk, "Phase Diagram of the System KLu(WO)-KNd(WO) and Growth of KLu(WO) Single Crystals," Inorganic Materials, Vol. 23, No. 11, 1987, pp. 1657-1660.

[9] J. X. Zhang, K. P. Wang, J. Y. Wang, H. J. Zhang, W. T. Yu, X. P. Wang, Z. P. Wang, Q. M. Lu and M. F. Ba, “Anisotropic Thermal Properties of Monoclinic Yb:KLu(WO) Crystals," Applied Physics Letters, Vol. 87, No. 6, 2005, Article ID 061104, pp. 1-3. 